\title{
Recall of two visual targets embedded in RSVP streams of distractors depends on their temporal and spatial relationship
}

\author{
SHUI-I SHIH \\ University of Southampton, Highfield, England
}

\begin{abstract}
In the present study, I examined how the temporal and spatial relationship between two visual targets (T1 and T2) affects the recall of both targets when they are embedded in rapidly displayed distractors. Presented on a trial were two synchronized streams of characters, one to the left and the other to the right of the fixation. Independent of their spatial relationship, a U-shaped curve described the recall of the second target (T2) as a function of stimulus onset asynchrony (SOA) between T1 and T2. It indicated the presence of the attentional blink with a T2 deficit sparing up to about 150- to 200-msec SOA. However, T2 deficit was greater at short SOAs (up to about $250 \mathrm{msec}$ ) when T1 and T2 occurred at different locations than when they occurred at a common location. When SOA was short (100 $\mathrm{msec}$ or so), recall of T1 was impaired when T1 and T2 occurred at a common location, but not when they were at different locations. The present findings can be reconciled with existing models (e.g., the interference model and the two-stage model) by distinguishing automatic and controlled attention gating processes at the transfer of perceptual representations to a more durable storage (e.g., visual short-term memory).
\end{abstract}

The ability to identify and remember visual events occurring in rapid succession sometimes plays an important role in daily life. For example, it affects the reliability of an eyewitness and the efficiency of an advertisement presented in animation. Such ability is typically studied by using the rapid serial visual presentation (RSVP) procedure. Let $\mathrm{T} 1$ and $\mathrm{T} 2$ be two visual events and $\mathrm{T} 1$ occur before $\mathrm{T} 2$ in time. When $\mathrm{T} 1$ and $\mathrm{T} 2$ are embedded in an RSVP stream of distractors, a recall deficit of T2 may be observed if the interval between $\mathrm{T} 1$ and $\mathrm{T} 2$ is within $500 \mathrm{msec}$ or so (e.g., Chun, 1997; Chun \& Potter, 1995; Grandison, Ghirardelli, \& Egeth, 1997; Maki, Couture, Frigen, \& Lien, 1997; Raymond, Shapiro, \& Arnell, 1992, 1995; Ward, Duncan, \& Shapiro, 1997). The deficit is attributed to an attentional blink.

An attentional blink is commonly referenced to the lag between T1 and T2. A condition of lag $n$ means that there are $n-1$ distractors between T1 and T2. Given a steady presentation rate (typically 10 items/sec), the lag $n$ also reflects the stimulus onset asynchrony (SOA) between T1 and T2. As a function of SOA (and lag), the recall of T2 can be described by two types of curve. Some studies have shown a monotonically increasing curve up to $700 \mathrm{msec}$ with maximum deficit of $\mathrm{T} 2$ recall at Lag 1 (e.g., Duncan, Martens, \& Ward, 1997; Joseph, Chun, \& Nakayama, 1997; Maki et al., 1997, Experiment 2), whereas other studies have shown a U-shaped curve with $\mathrm{T} 2$ report at Lag 1

S.-I S. thanks Marvin Chun, Steve Yantis, and two anonymous reviewers for informative comments and suggestions. Correspondence should be addressed to S.-I Shih, Department of Psychology, University of Southhampton, Highfield S017 1BJ, England (e-mail: sis@, soton.ac.uk). relatively unimpaired (e.g., Chun \& Potter, 1995; Raymond et al., 1992; Seiffert \& Di Lollo, 1997; Vogel, Luck, \& Shapiro, 1998). Lag 1 sparing is used to refer to the relatively unimpaired T2 report at Lag 1 (Potter, Chun, Banks, \& Muckenhoupt, 1998). After reviewing more than 100 experiments of attentional blinks, Visser, Bischof, and Di Lollo (1999) concluded that Lag 1 sparing is independent of attentional blink and its occurrence depends on the nature of attention switch involved in the task: Lag 1 sparing occurs when no attention switch is required between the targets (e.g., identify 2 digits among letters at the fixation) or when the switch involves only one dimension (e.g., a task switch: identify $\mathrm{T} 1$ and detect T2). Lag 1 sparing is absent when the switch involves location (i.e., $\mathrm{T} 1$ and T2 occur at different locations) or with more than one dimension (e.g., identify an auditory $\mathrm{T} 1$ and detect a visual T2).

In the present study, I examined how the temporal and spatial relationship between two visual targets embedded in streams of distractors affects the processing of both targets when allocation of spatial attention is controlled. Most studies of attentional blinks have presented all stimuli in a single spatial location - namely, the fixated area. Although models of attentional blinks do not explicitly address whether the deficit is location (or channel) specific, they have assumed a central limitation in consolidating perceptual information (e.g., the interference model, Shapiro, Raymond, \& Arnell, 1994; the two-stage model, Chun \& Potter, 1995). Hence, the attentional blink would be expected regardless of the spatial relationship between the two targets. If the deficit depended on their spatial relationship, it would suggest a contribution of a processing limitation in an earlier (e.g., perceptual) stage. 
Indeed, several studies have revealed attentional blinks when $\mathrm{T} 1$ and $\mathrm{T} 2$ occur at different spatial locations (e.g., Duncan et al., 1997; Duncan, Ward, \& Shapiro, 1994; Joseph et al., 1997; Ward, Duncan, \& Shapiro, 1996). However, these studies did not permit direct evaluation of how the deficit would vary with the spatial relationship between $\mathrm{T} 1$ and $\mathrm{T} 2$ because they were always presented at different locations. Moreover, the potential shift of attention from one location to another has rendered these studies unsuitable to directly answer the present concern. For example, Duncan et al. (1997) revealed the attentional blink without Lag 1 sparing when using four RSVP streams consisting of strings of $\mathrm{xxx}$ and two targets. One of the targets was always randomly presented at one of the two horizontal streams (i.e., 3 and 9 o'clock positions) and the other at one of the two vertical streams. The horizontal streams alternated with the vertical streams with a brief overlapping interval. Shih and Sperling (1996) demonstrated that observers were capable of shifting attention to different locations from frame to frame in an RSVP stream displayed at an SOA of about 135-200 msec when the likely target itself provided the cue to its spatial location (e.g., each frame contained one red item and several green items). The unsynchronized presentation in Duncan et al. (1997) allowed the observers to alternately attend to horizontal and vertical locations. The potential covert attention shift might have contaminated the results.

Ward et al. (1996, which includes Duncan et al., 1994) used the same stimulus layout and presentation constraints as Duncan et al. (1997). However, instead of embedding T1 and T2 in RSVP streams, the visual presentation included only four events: T1, T1-mask, T2, and T2-mask. Although their observers were likely to use a divided/diffused attention mode because $\mathrm{T} 1$ and $\mathrm{T} 2$ could be presented simultaneously or with a very short interval (e.g., SOA $=100 \mathrm{msec}$ ), the attentional blink was revealed with an absence of Lag 1 sparing. Its absence can be attributed to the phenomenon of attentional capture. In their procedure, $\mathrm{T} 1$ was clearly an abrupt-onset object among the stationary place markers and fixation point. It has been well established that a salient and a new perceptual object summons attention involuntarily when the observers are in a diffused attention mode (e.g., Bacon \& Egeth, 1994; Folk, Remington, \& Johnston, 1992; Jonides \& Yantis, 1988; Theeuwes, 1994; Yantis \& Jonides, 1984). In other words, in Ward et al., attention might have been involuntarily directed to T1. Moreover, as Moore, Egeth, Berglan, and Luck (1996) have pointed out, a postmasked T1 might have engaged attention for a longer time than an unmasked T1. Consequently, when the SOA was short, attention might not have been available to a new location in which T2 was presented, and hence a deficit was revealed. It is also possible that the T1-mask had some masking effect on T2 at short SOA (i.e., 0-300 msec) conditions in which $\mathrm{T} 2$ was displayed at the presence of the T1-mask. In sum, the recall deficit of T2 observed in Ward et al. might not have been a simple result of the attentional blink.

The discussions above are consistent with Visser et al.'s (1999) conclusion: "Lag 1 sparing is never found following a switch in location, whether implemented alone or in conjunction with a switch in another dimension" (p. 464). However, Visser et al. suggested that when T1 and $\mathrm{T} 2$ occur at different locations, the $\mathrm{T} 2$ at Lag 1 could still be spared from the deficit if attention is distributed to the relevant locations. In support of this contention, they cited studies of Sperling (1960) and Peterson and Juola (1997). Although Sperling's experiments on iconic memory required observers to attend to several locations at the same time (if possible), the stimulus was typically a singly presented array of a dozen or so characters, not an RSVP stream of characters. In Peterson and Juola, participants had to use a strategy of attention switch because the location of $\mathrm{T} 2$ was cued by $\mathrm{T} 1$ when they occurred at different locations. Hence, neither of them provided direct evidence in the present context. The present study was intended to fill this gap in the literature.

The present experiment was intended to discourage a processing strategy involving attention switch/shift while investigating the effect of spatial and temporal relationship between two visual targets on the recall of both targets when they are embedded in two RSVP streams of characters. To prevent involuntary attentional shifts (i.e., attentional capture), two synchronized RSVP streams of characters, at the 3 and 9 o'clock positions, were presented on each trial. The spatial location of T1 (left or right) and the spatial location of $\mathrm{T} 2$ were crossed. To discourage voluntary attentional shifts, the probability of $\mathrm{T} 1$ and $\mathrm{T} 2$ being presented at the same spatial location was equal to the probability of their being presented at different locations. Hence, the best strategy was to maintain the mode of divided attention and to monitor the two streams at the same time.

The two targets were sampled from the same conceptual category (i.e., digit) without any other distinctive stimulus feature (e.g., color or brightness) to distinguish between them and the distractor letters. The participants were asked to perform an identification-and-identification rather than identification-and-detection dual task. The advantages of using a dual identification-and-identification task are twofold: (1) It does not involve a task switch, and (2) every trial contributes to data analysis. ${ }^{1}$

\section{METHOD}

\section{Participants}

There were 20 volunteers with normal or corrected-to-normal vision. Each was paid $£ 2$ for a 45 -min session. The distance between an observer and visual presentations on a CRT screen was about $70 \mathrm{~cm}$ and was maintained with the aid of a chinrest.

\section{Stimuli}

The targets were randomly selected from 9 digits - $1,2,3,4,5$, $6,7,8$, and 9 . The distractors were randomly selected from a set of 
16 uppercase letters-A, C, D, E, F, H, K, L, M, N, T, U, V, W, X, and $Y$. The width and height of the largest letter $M$ were about $0.90^{\circ}$ and $0.74^{\circ}$ of visual angle (dva). A square of $0.25 \times 0.25 \mathrm{dva}^{2}$ was used as a fixation point throughout a trial. An SVGA monitor with a refresh rate of $70 \mathrm{~Hz}$ was used to present stimuli. Stimuli appeared white $\left(33 \mathrm{~cd} / \mathrm{m}^{2}\right)$ on a dark background $\left(9.1 \mathrm{~cd} / \mathrm{m}^{2}\right)$. The experimental cubicle was dimly lit.

\section{Design and Procedure}

There were three independent variables. (1) The lag between the two targets ( $\mathrm{T} 1$ and $\mathrm{T} 2$ ) was varied between 0 and 8 , yielding an SOA between 0 and $571 \mathrm{msec}$. (2) The spatial location of T1 was either left or right. (3) The congruency in spatial location between $\mathrm{T} 1$ and $\mathrm{T} 2$ was either congruent (same location) or incongruent (different locations). The three variables were crossed, yielding 34 (i.e., $2+8 \times 2 \times 2$ ) conditions because the condition of Lag 0 was possible only when $\mathrm{T} 1$ and $\mathrm{T} 2$ occurred in different locations. There were a total of 408 trials, 12 for each condition, in one experimental session. The presentation order of conditions was completely randomized. The experimental trials were preceded by 12 practice trials with randomly selected conditions.

Two synchronized streams of characters were presented on each trial - one stream to the left of the fixation and the other to the right. The center-to-center distance between the two streams was about 3.5 dva. There were $14-28$ letters in a stream. The two digits in a trial were of different identity and could occur in congruent or incongruent locations. The number of letters preceding $\mathrm{T} 1$ was varied between 8 and 14 , with probabilities determined by the function $P(n)=2 \times(1 / 3)^{(n-7)}, n$ in $(8,14)$, so that the expectation of T1's occurrence remained constant across the seven locations. There were always 13 letters and 1 digit subsequent to $\mathrm{T} 1$ in the locationcongruent condition, and 14 letters in the incongruent condition. Each stimulus frame (consisting of two characters) was presented for one refresh (i.e., 1/70 sec) and followed by four refreshes of blank frame. Thus, SOA was about $71.4 \mathrm{msec}$. The fixation square remained present throughout a trial.

Each trial began with a 157-msec (i.e., 11 refreshes) fixation display, which was immediately followed by RSVP stimulus streams. At the end of each trial, the observer was first prompted with "First?" on the screen to enter the identity of the digit which he/she believed or guessed to have been presented first (T1), and then "Second?" to enter the identity of the second digit (T2). The observer must enter a valid response (i.e., 1-9) for the experiment to proceed. The experiment was self-paced, and the observers were encouraged to take breaks whenever they needed to.

\section{RESULTS}

The data of 1 participant were excluded from the analysis because he/she gave the same report for both targets in a large proportion of trials. This left the data of 19 participants in the following analysis.

\section{Recall Accuracy for the Simultaneously Presented Targets}

At Lag 0, mean recall accuracy for the target presented on the left $(0.84)$ was significantly higher than that on the right $(0.67)$ [paired $t(18)=4.7, p<.001$ ]. When the left target was successfully reported, the mean recall accuracy for the right target was 0.80 . However, when the right target was successfully reported, the mean recall accuracy for the left target was only 0.64 . Such a discrepancy suggested that observers might have been biased toward the left location either by fixating at a location toward to the left or by paying more attention to the left due to a life-long reading habit. In fact, at Lag 0 , the left target was usually reported before the right target. Given correct recall for the left target, the chance of reporting the left target first was about $76 \%$, whereas given correct recall for the right target, the chance of reporting the right target first was only about $30 \%$.

The following analyses focused on the recall accuracy of $\mathrm{T} 2$ conditioned on correct $\mathrm{T} 1$ report $(P(\mathrm{~T} 2 \mid \mathrm{T} 1))$ and recall accuracy of $\mathrm{T} 1$ conditioned on correct $\mathrm{T} 2$ report $(P(\mathrm{~T} 1 \mid \mathrm{T} 2))$ because the main purpose was to examine how the processing of $\mathrm{T} 1$ and $\mathrm{T} 2$ influences each other. It was not unusual that $\mathrm{T} 1$ and $\mathrm{T} 2$ were not recalled in the order as they had been presented. For example, T2 might be reported as $\mathrm{T} 1$ if $\mathrm{T} 1$ was missed; $\mathrm{T} 1$ and $\mathrm{T} 2$ might be reported in a reversed order when the lag was small (see below). However, preliminary analyses showed that the result pattern for $P(\mathrm{~T} 2 \mid \mathrm{T} 1)$ and $P(\mathrm{~T} 1 \mid \mathrm{T} 2)$, respectively, did not depend on whether the accuracy in report order was taken into account. Hence, the accuracy in report order was not incorporated into the dependent measures.

\section{Conditional Identity Accuracy of T2 Given Accurate T1 Identity, $P(T 2 \mid T 1)$}

$P(\mathrm{~T} 2 \mid \mathrm{T} 1)$ s were analyzed in a three-way analysis of variance (ANOVA) with within-subjects factors of lag, T1 location, and location congruency (LC) between $\mathrm{Tl}$ and $\mathrm{T} 2$. There were significant main effects of $\operatorname{lag}[F(7,126)=$ $19.1, p<.001], \mathrm{T} 1$ location $[F(1,18)=13.1, p \approx .002]$, and $\operatorname{LC}[F(1,18)=7.5, p \approx .01]$, and significant interactions of $\mathrm{LC} \times \operatorname{lag}[F(7,126)=8.9, p<.001]$ and $\mathrm{LC} \times$ T1 location $[F(1,18)=39.3, p<.001]$. Because the threeway interaction was nonsignificant $(p \approx .2), P(\mathrm{~T} 2 \mid \mathrm{T} 1) \mathrm{s}$ were collapsed across the levels of T1 location. Figure $1 \mathrm{~A}$ presents mean $P(\mathrm{~T} 2 \mid \mathrm{T} 1)$ as a function of lag (SOA) with $\mathrm{LC}$ as the curve parameter. The occurrence of attentional blinks was indicated by the significantly U-shaped curves [congruent, $F(1,18)=54.7, p<.001$; incongruent, $F(1,18)=73.8, p<.001]$. Relative to the LC condition, the recall deficit of T2 was higher (a cost of about $20 \%$ ) in the location-incongruent condition when the SOA between $\mathrm{T} 1$ and $\mathrm{T} 2$ was short (i.e., less than about $250 \mathrm{msec}$ ). Such a difference was significant at Lags 1, 2 , and 3 [paired $t(18)=5.7,4.2,5.1$, respectively, all $p \mathrm{~s}<.001]$. However, the difference disappeared from Lag $4(\mathrm{SOA}=286 \mathrm{msec})$ onward (all $p \mathrm{~s}>.05$ in paired $t$ tests). The sparing of $\mathrm{T} 2$ recall deficit was evaluated by pairwise comparisons with Bonferroni adjustment using the lowest $P(\mathrm{~T} 2 \mid \mathrm{T} 1)$ at Lag 5 as the reference level. For the location-incongruent condition, the sparing was significant at Lag $1(p \approx .01)$. For the location-congruent condition, the sparing was significant at Lag $1(p<$ $.001)$, Lag $2(p<.001)$, and Lag $3(p \approx .003)$. The extended sparing could be due to a faster presentation rate (about $14 \mathrm{items} / \mathrm{sec}$, whereas a conventional rate is about 10 items $/ \mathrm{sec}$ ). 

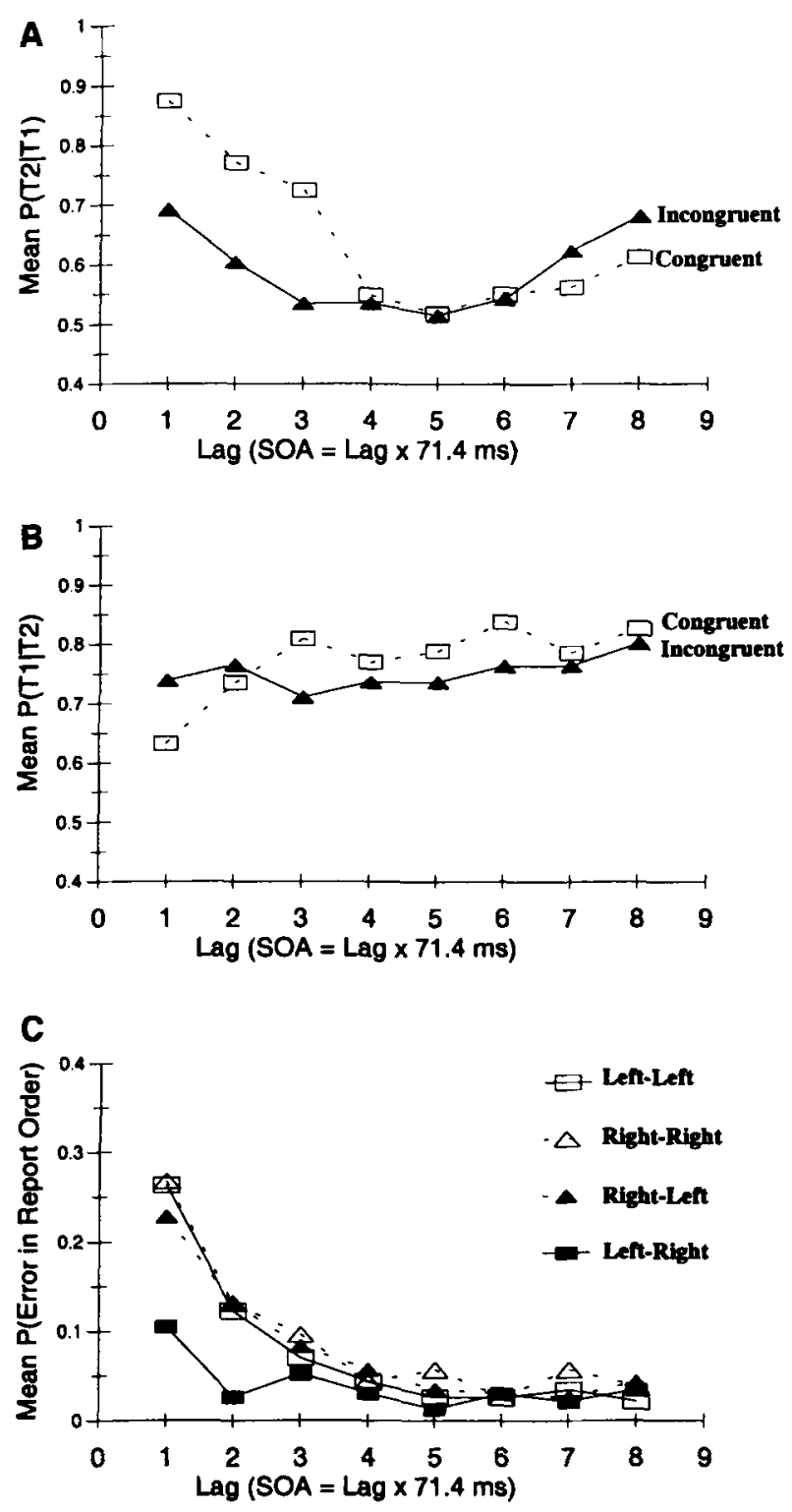

Figure 1. A-B: Mean report accuracy of one target (T1 or T2) given the correct report of the other target ( $T 2$ or $\mathrm{T} 1$ ) as a function of lag (stimulus onset asynchrony [SOA]) with location congruency between the two targets as the curve parameter. $C$ : Mean proportion of reporting the two targets in reverse order as a function of lag (SOA) with spatial relationship as the curve parameter-T1 location is hyphenated with $\mathrm{T} 2$ location.

\section{Conditional Identity Accuracy of}

\section{T1 Given Accurate T2 Identity, P(T1|T2)}

$P(\mathrm{~T} 1 \mid \mathrm{T} 2)$ s were analyzed by a three-way ANOVA with within-subjects factors of lag, T1 location, and LC between $\mathrm{T} 1$ and $\mathrm{T} 2$. The data of 1 participant were excluded from the analysis because of missing values [i.e., $P(\mathrm{~T} 1 \mid \mathrm{T} 2)$ is undefined when $P(\mathrm{~T} 2)=0]$. There were significant main effects of lag $[F(7,119)=4.9, p<.001]$ and $\mathrm{T} 1$ location $[F(1,17)=11.8, p \approx .003]$ and a significant interaction of lag $\times \operatorname{LC}[F(7,119)=2.93, p \approx .007]$.
Because the three-way interaction was nonsignificant $(p \approx .2), P(\mathrm{~T} 1 \mid \mathrm{T} 2)$ s were collapsed across the levels of $\mathrm{T} 1$ location. Figure $1 \mathrm{~B}$ presents mean $P(\mathrm{~T} 1 \mid \mathrm{T} 2)$ as a function of lag (SOA) with LC as the curve parameter. In the location-incongruent condition, $P(\mathrm{~T} 1 \mid \mathrm{T} 2)$ did not vary with lag. In the location-congruent condition, by contrast, there was a significant quadratic trend in lag, with $P(\mathrm{~T} 1 \mid \mathrm{T} 2)$ lowest at Lag 1 and asymptoting at Lag 3 $[F(1,18)=10.31, p=.005]$.

\section{Error in Report Order}

Consider the trials in which both T1 and T2 were reported. Figure $1 \mathrm{C}$ presents the proportion of reporting the two targets in a reverse order with spatial locations of $\mathrm{T} 1$ and $\mathrm{T} 2$ as the curve parameter. Except for the condition in which $\mathrm{T} 1$ was presented at the left while $\mathrm{T} 2$ was presented at the right (left-right), the results for the remaining conditions were similar to each other. That is, the error was about $25 \%$ at Lag 1, it dropped to $13 \%$ at Lag 2, and from Lag 3, the error remained at about $7 \%$. These results replicated the typical findings that the temporal orders of two targets were poorly recorded when they occurred close in time (e.g., within $200 \mathrm{msec}$, Chun \& Potter, 1995; Raymond et al., 1992; Reeves \& Sperling, 1986). The difference in the error between the left-right and the remaining conditions was primarily due to a lower error rate at Lag $1(10 \%)$ and Lag $2(3 \%)$ in the left-right condition. As indicated in the above analysis, the participants had a tendency to report the left target first, regardless of presentation order. Such a tendency accounted for the unusually low error rate in report order at short lag/SOAs for the left-right condition.

\section{DISCUSSION}

The present study has shown that attentional blinks and Lag 1 sparing are independent of the spatial relationship between the two visual target events (T1 and T2). Such an independence supports the contention that the attentional blink is due to a limitation at a more central processing stage in which inputs from space-dependent channels (e.g., fovea vs. peripheral retina area) compete for space-independent processing resources. For example, the interference model conceptualized such space-independent resources as attentional weightings in visual short-term memory, whereas the two-stage model regarded these resources as being used in a process of identifying the potential target and consolidating it for later report. The present findings also provide direct support for Visser et al.'s (1999) contention that T2 occurring at short lags can be spared from the deficit "when a task requires the identification of multiple stimuli within a larger attended area" (p. 464).

However, two kinds of space-dependent performance difference revealed in the present findings deserve further consideration. First, $P(\mathrm{~T} 2 \mid \mathrm{T} 1)$ was about $20 \%$ higher in the location-congruent condition than in the locationincongruent condition when the SOA between $\mathrm{T} 1$ and $\mathrm{T} 2$ 
was less than $250 \mathrm{msec}$. Second, $P(\mathrm{~T} 1 \mid \mathrm{T} 2)$ was independent of lag in the location-incongruent condition, but it was about $10 \%$ lower at $\mathrm{Lag} 1$ (SOA $\approx 71 \mathrm{msec}$ ) in the location-congruent condition. Similar results were revealed in Chun and Potter (1995, Experiment 1), which involved only one spatial location: at Lag 1 (SOA = $100 \mathrm{msec}), \mathrm{T} 2$ was better recalled than T1 ( $82 \%$ vs. $67 \%)$. Unlike in most of the related studies, Tl was not associated with a salient physical feature (e.g., color) in both Chun and Potter and the present studies. It seems that associating $\mathrm{T} 1$ with a salient feature can spare $\mathrm{T} 1$ from deficit related to the arrival time of $\mathrm{T} 2$. However, without a salient feature associated with $\mathrm{T} 1$, a deficit of $\mathrm{T} 1$ report at Lag 1 was revealed when T1 and T2 had been presented at a common location, but not when they had been presented at different locations. Apparently, there was a tradeoff between reports of T1 and T2 at Lag 1 when a salient feature was absent in $\mathrm{T} 1$ in location-congruent condition. Because the existing theories of attentional blink do not explicitly predict space-dependent variation in performance and do not specify the possible role of a salient feature, the present findings cannot be fully explained by these theories. However, incorporating a "peripheral" attentional mechanism (Weichselgartner \& Sperling, 1987) could reconcile the present results with these theories.

\section{Peripheral Attentional Processes}

Weichselgartner and Sperling (1987) presented to the observer an RSVP sequence of digits at the fixation. They found a bimodal distribution of recall probability as a function of SOA when the target was distinctive (e.g., using a bright outline square to cue the target). The first mode occurred at SOAs between 0 and $100 \mathrm{msec}$, indicating that the target and the digit immediately subsequent to the target were reported with high accuracy $(70 \%-$ $90 \%$ ). The second mode (with a peak of about $60 \%$ ) occurred at SOAs between 300 and $400 \mathrm{msec}$. They also showed that the second mode (but not the first mode) was dependent on "factors that classically affect task difficulty: practice, expectation, stimulus probability in mixed-list designs, and target signal-to-noise ratio" (p. 780). For example, using a dim outline square delayed the peak of the second mode by about $100 \mathrm{msec}$, but it did not affect the first mode. However, using a very dim but detectable outline square to cue the target resulted in a loss of the first mode and a broad distribution of the recall probability with peak (at about $60 \%$ ) spanning from 100 to $500 \mathrm{msec}$ after the target onset. Hence, they suggested that the first mode represents a fast, automatic process and the second mode a slower, effortful, controlled process, and that the two processes could be partially concurrent.

These processes are regarded as a "peripheral" attentional mechanism because they characterize the transfer of perceptual representations (i.e., the contents of iconic memory or sensory visual memory) to visual short-term memory. Reeves and Sperling (1986; see also Sperling \&
Weichselgartner, 1995) called such a transfer process attention gating. In fact, such a peripheral attentional mechanism was hinted at in the theories of attentional blinks. Both interference and two-stage models proposed that T1 gained access to visual short-term memory because of its good match to the target's template. They also proposed that the item immediately subsequent to the target gained access to visual short-term memory because the presentation rate exceeded the temporal resolution (i.e., $\mathrm{SOA} \leq$ $100 \mathrm{msec}$ ) of the relevant mental process. "Gaining access to visual short-term memory" is a concept equivalent to an attention gating function.

\section{Attention Gating: Transfer \\ Perceptual Representations to VSTM}

The basic concepts of attention gating are illustrated in Figure 2. Because all the items in a given RSVP sequence had the same presentation condition and duration, the actual form of the function relating perceptual availability to time is immaterial to the present discussion. Hence, a rectangular function with a width equal to the SOA was arbitrarily chosen to represent the perceptual availability of an item: An item is immediately available at its onset and persists until the onset of the next item. Figure 2A presents a sequence of perceptual representations of RSVP items presented at a common spatial location. Due to equal strength in perceptual representations, the strength of an item's representation in visual short-term memory depends on the attention gating function. Figure $2 \mathrm{~B}$ sketches a hypothetical attention gating function for that location. Figure $2 \mathrm{C}$ illustrates the function of attention-gated representations: the product of Figures $2 \mathrm{~A}$ and $2 \mathrm{~B}$. The vertical lines in Figures $2 \mathrm{~A}$ and $2 \mathrm{C}$ indicate the separation between two successive items. For each item, the relative area under the curve (i.e., the integration of the product of Figures $2 \mathrm{~A}$ and $2 \mathrm{~B}$ ) indicates its relative strength in visual short-term memory. An item is not represented in visual short-term memory (and hence cannot be reported later except by chance error) if its perceptual representation exists before the gate opens or after the gate closes.

\section{Automatic Attention Gating}

In the conventional procedure for investigating attentional blinks, $\mathrm{T} 1$ is typically distinctive (e.g., white T1 among black items) and hence can trigger an automatic process. For attention gating, an automatic process means a fast opening and closing rate and a fixed interval/width of the gate. The results of Weichselgartner and Sperling (1987, Figures $2 \mathrm{~b}$ and $2 \mathrm{c}$ ) indicate that the width of an automatic attention gating is about $100-150 \mathrm{msec}^{2}$ An example of automatic attention gating is sketched in Figures $2 \mathrm{~B}$ and $2 \mathrm{C}$, in which the strength of $\mathrm{T} 1$ represented in visual short-term memory (i.e., the area under its representation function) is greater than that of the subsequent T2 presented at the same spatial location. Given that the match of T2 to its template is about as good as the match of $\mathrm{Tl}$ to its template, such an automatic process ensures 


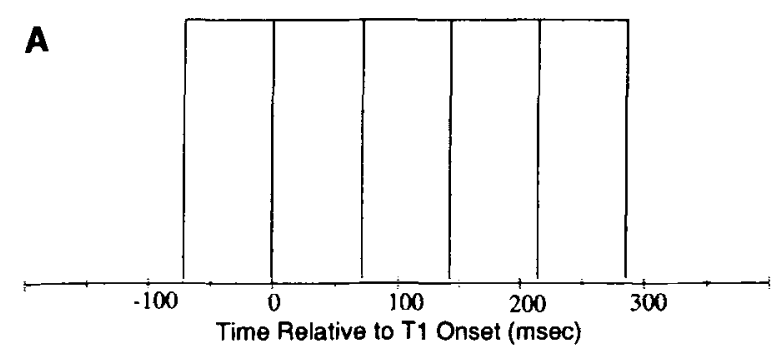

Automatic Gating
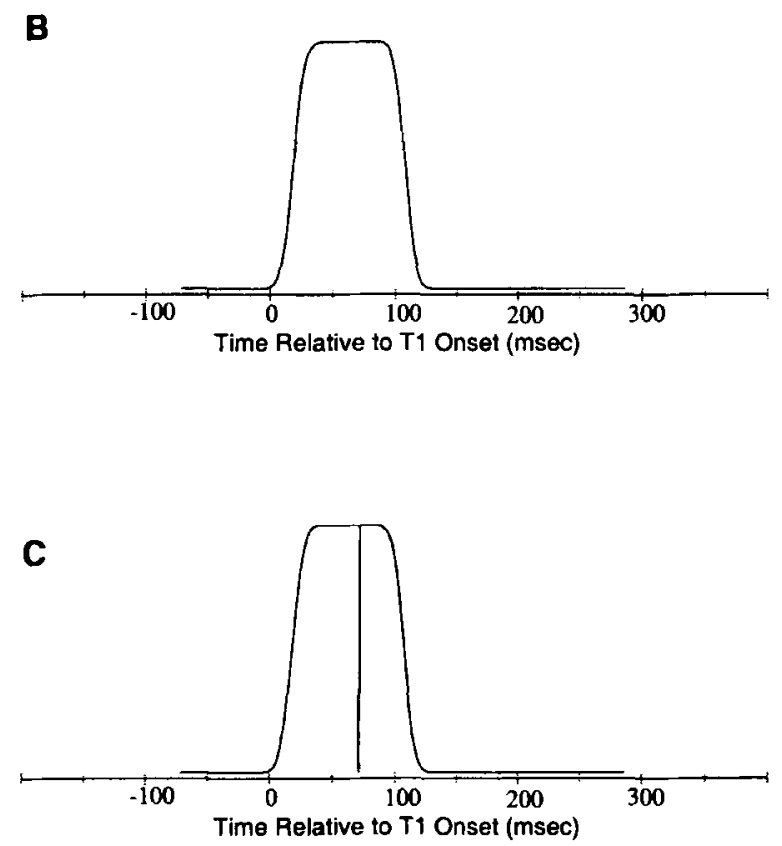

\section{Controlled Gating}
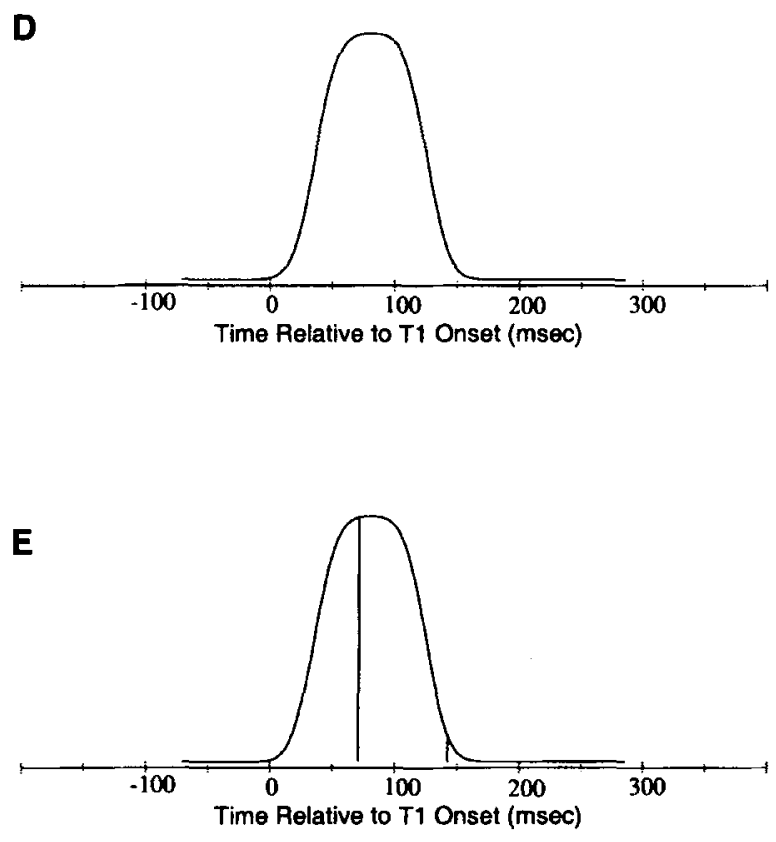

Figure 2. Attention gating. A: A series of perceptual representations, each one available from its onset to the onset of the subsequent item. The onset of T1 is designated as the Time 0 . B: An automatic attention gate with fast opening/closing rate and a width of 125 msec. C: Representations in a durable storage (e.g., visual short-term memory) that are transferred from perceptual representations via the attention gate- the product of $A$ and $B$. D: $A$ controlled attention gate with an opening/closing rate slower than that in $B$ and a width the same as that in B. E: Representations in a durable storage-the product of $A$ and $D$. The vertical lines in $A, C$, and $E$ indicate the separation between two successive items.

a processing priority for $\mathrm{T} 1$ and results in a consistently high accuracy for $\mathrm{T} 1$ report.

\section{Controlled Attention Gating}

In the present study there was no unique feature (e.g., higher luminance, an odd color, or an outlined square) associated with $\mathrm{T} 1$. As a result, automatic attention gating was unlikely to occur. Instead, on the detection of $\mathrm{T} 1$, a controlled attention gating took place. A controlled process in attention gating means an opening and closing rate slower than that for automatic attention gating and a variable width of the gate depending on the task and the strategy of the participants. With the same gate width as that shown in Figure 2B but a slower opening/closing rate, an example of controlled attention gating is sketched in Figures $2 \mathrm{D}$ and $2 \mathrm{E}$, in which the strength of $\mathrm{T} 1$ repre- sented in visual short-term memory is lower than that of the subsequent $\mathrm{T} 2$ presented at the same spatial location. Given that the match of T2 to its template is about as good as the match of $\mathrm{T} 1$ to its template, such a controlled process gives a lower processing priority to $\mathrm{T} 1$ than to $\mathrm{T} 2$. This results in a report accuracy of $\mathrm{T} 1$ at Lag 1 lower than that at other lags.

Although controlled attention gating can take place at several spatial locations at the same time (Shih, 1992; Sperling \& Weichselgartner, 1995), there is typically a delay between two consecutive gating operations occurring at different locations. However, the delay is independent of the distance between the two locations (Sperling \& Weichselgartner, 1995). Hence, when T1 and T2 were presented at different locations, the strength of $\mathrm{T} 2$ represented in visual short-term memory was at most as 
strong as (and most likely weaker than) that of T1. Therefore, it is reasonable to expect that when $\mathrm{T} 1$ and $\mathrm{T} 2 \mathrm{oc}-$ curred at different spatial locations, the processing of $\mathrm{T} 1$ would be relatively independent of the timing of T2's arrival. This would account for the lack of a lag effect for $\mathrm{T} 1$ report in the location-incongruent condition. Because of the delay, we would expect greater impairment for $\mathrm{T} 2$ report at short lags in the location-incongruent condition. However, the greater impairment would disappear by the time at which processing of $\mathrm{T} 1 \mathrm{had}$ been completed. On the departure of $\mathrm{Tl}$ to another stage, the resources initially attached to $\mathrm{T} 1$ would be released so that further processing disadvantage for $\mathrm{T} 2$ would be eliminated.

In sum, the present findings do not contradict the existing theories of attentional blinks-namely, the interference model and the two-stage model. However, the present results suggest an elaboration for these models with regard to the admission of a perceptual representation to visual short-term memory. Although the role of attention in transferring perceptual representation of a stimulus item to visual short-term memory has been implicated in both models, neither model has explicitly considered the operational characteristics of such "peripheral" attentional mechanisms.

\section{Summary}

In the present study, the spatial relationship between two visual target events (T1 and T2) was manipulated and the use of attention switch was discouraged. Attentional blinks and Lag 1 sparing were revealed to be independent of the spatial relationship between $\mathrm{T} 1$ and $\mathrm{T} 2$. Theoretically, the present study supports the contention that the attentional blink reflects the limitation at a higher level of processing, for a low-level limitation (e.g., perceptual processing or iconic memory) tends to be location specific. However, the present results also showed that a peripheral operation of attention gating could modulate the processing of $\mathrm{T} 1$ and $\mathrm{T} 2$ in the paradigm of attentional blinks. The peripheral attention gating transfers perceptual representations to visual short-term memory (Reeves \& Sperling, 1986; Weichselgartner \& Sperling, 1987). A salient $\mathrm{T} 1$ could initiate an automatic attention gating ensuring processing priority for $\mathrm{T} 1$ and resulting in an absence of lag effect for $\mathrm{T} 1$. In the absence of a distinctive feature, controlled attention gating with slower opening/ closing rate than that of automatic gating could account for the observed impairment of $\mathrm{T} 1$ report at short lags.

\section{REFERENCES}

Bacon, W. F., \& EgETh, H. E. (1994). Overriding stimulus-driven attentional capture. Perception \& Psychophysics, 55, 485-496.

Chun, M. M. (1997). Types and tokens in visual processing: A double dissociation between the attentional blink and repetition blindness. Journal of Experimental Psychology: Human Perception \& Performance, 23, 738-755.

Chun, M. M., \& Potter, M. C. (1995). A two-stage model for multiple target detection in rapid serial visual presentation. Journal of Experimental Psychology: Human Perception \& Performance, 21, 109127.
Duncan, J., Martens, S., \& Ward, R. (1997). Restricted attentional capacity within but not between sensory modalities. Nature, $\mathbf{3 8 7}$, 808-810.

DunCan, J., Ward, R., \& Shapiro, K. (1994). Direct measurement of attentional dwell time in human vision. Nature, 369, 313-315.

Folk, C. L., Remington, R. W., \& Johnston, J. C. (1992). Involuntary covert orienting is contingent on attentional control settings. Journal of Experimental Psychology: Human Perception \& Performance, 18, 1030-1044.

Grandison, T. D., Ghirardelli, T. G., \& Egeth, H. E. (1997). Beyond similarity: Masking of the target is sufficient to cause the attentional blink. Perception \& Psychophysics, 59, 266-274.

JONIDES, J., \& YANTIS, S. (1988). Uniqueness of abrupt visual onset in capturing attention. Perception \& Psychophysics, 43, 346-354.

JosePh, J. S., Chun, M. M., \& NaKayama, K. (1997). Attentional requirements in a 'preattentive' feature search task. Nature, 387, 805807.

Maki, W. S., Couture, T., Frigen, K., \& Lien, D. (1997). Sources of the attentional blink during rapid serial visual presentation: Perceptual interference and retrieval competition. Journal of Experimental Psychology: Human Perception \& Performance, 23, 1393-1411.

Moore, C. M., Egeth, H. E., Berglan, L. R., \& Luck, S. J. (1996). Are attentional dwell times inconsistent with serial visual search? $P s y$ chonomic Bulletin \& Review, 3, 360-365.

Peterson, M. S., \& Juola, J. F. (1997, November). Spatial and temporal dynamics of attentional gating and the attentional blink. Poster presented at the 38th annual meeting of the Psychonomic Society, Philadelphia.

Potter, M. C., Chun, M. M., Banks, B. S., \& Muckenhoupt, M (1998). Two attentional deficits in serial target search: The visual attentional blink and an amodal task-switch deficit. Journal of Experimental Psychology: Learning, Memory, \& Cognition, 24, 979-992.

Raymond, J. E., Shapiro, K. L., \& Arnell, K. M. (1992). Temporary suppression of visual processing in an RSVP task: An attentional blink? Journal of Experimental Psychology: Human Perception \& Performance, 18, 849-860.

Raymond, J. E., Shapiro, K. L., \& Arnell, K. M. (1995). Similarity determines the attentional blink. Journal of Experimental Psychology: Human Perception \& Performance, 21, 653-662.

REEVES, A., \& SPERLING, G. (1986). Attention gating in short-term visual memory. Psychological Review, 93, 180-206.

SeifferT, A. E., \& Di Lollo, V. (1997). Low-level masking in the attentional blink. Journal of Experimental Psychology: Human Perception \& Performance, 23, 1061-1073.

Shapiro, K. L., Raymond, J. E., \& ArNell, K. M. (1994). Attention to visual pattern information produces the attentional blink in RSVP. Journal of Experimental Psychology: Human Perception \& Performance, 20, 357-371.

SHIH, S. (1992). Attentional components in early visual processing: A strength model of visual selective attention. Unpublished doctoral dissertation, New York University.

SHIH, S., \& SPERLING, G. (1996). Is there feature-based attentional selection in visual search? Journal of Experimental Psychology: Human Perception \& Performance, 22, 758-779.

SPERLING, G. (1960). The information available in brief visual presentations. Psychological Monographs, 74 (11, Whole No. 498).

SPERLiNG, G., \& WeichSElgarTnER, E. (1995). Episodic theory of the dynamics of spatial attention. Psychological Review, 102, 503-532.

TheEUWES, J. (1994). Stimulus-driven capture and attentional set: Selective search for color and visual abrupt onsets. Journal of Experimental Psychology: Human Perception \& Performance, 20, 799-806.

Visser, T. A. W., Bischof, W. F., \& Di Lollo, V. (1999). Attentional switching in spatial and nonspatial domains: Evidence from the attentional blink. Psychological Bulletin, 125, 458-469.

Vogel, E. K., LuCK, S. J., \& ShaPIRo, K. L. (1998). Electrophysiological evidence for a postperceptual locus of suppression during the attentional blink. Journal of Experimental Psychology: Human Perception \& Performance, 24, 1656-1674.

Ward, R., DunCan, J., \& ShaPIRo, K. (1996). The slow time-course of visual attention. Cognitive Psychology, 30, 79-109.

Ward, R., DunCan, J., \& Shapiro, K. (1997). Effects of similarity, dif- 
ficulty, and nontarget presentation on the time course of visual attention. Perception \& Psychophysics, 59, 593-600.

Weichselgartner, E., \& SPERLing, G. (1987). Dynamics of automatic and controlled visual attention. Science, 238, 778-780.

YANTIS, S., \& JoNidES, J. (1984). Abrupt visual onsets and selective attention: Evidence from visual search. Journal of Experimental Psychology: Human Perception \& Performance, 10, 601-621.

\section{NOTES}

1. Using a conventional dual identification-and-detection task (e.g., Raymond et al., 1992), half of the trials (with no probe or T2) were used for the purpose of satisfying a detection task and were usually not used for data analysis (but see Raymond et al., 1995, for sensitivity measures).

2. This judgment was based on the variation of recall probability given an SOA of $100 \mathrm{msec}$ : Accuracy was highest for the target (about $95 \%$ ), dropped $10 \%-20 \%$ for the next item, then fell to the minimum at the second item after the target.

(Manuscript received April 29, 1999; revision accepted for publication December 17, 1999.) 